\title{
Service-Based Survey of Dystonia in Munich
}

\author{
E. Castelon Konkiewitz ${ }^{\mathrm{a}} \quad$ I. Trender-Gerharda ${ }^{\mathrm{a}}$ C. Kamm ${ }^{\mathrm{b}}$ T. Warner \\ Y. Ben-Shlomo ${ }^{c}$ T. Gasserb ${ }^{b}$ B. Conrada A.O. Ceballos-Baumanna
}

Departments of a Neurology, Klinikum rechts der Isar, Technische Universität München, and ' ${ }^{\text {Neurology, }}$ Klinikum Grosshadern, Ludwig-Maximilians Universität München, Munich, Germany, and

CThe Epidemiological Study of Dystonia in Europe (ESDE) Collaborative Group

\author{
Key Words \\ Dystonia - Prevalence - Botulinum toxin · Epidemiology · \\ Munich
}

\begin{abstract}
We performed a service-based epidemiological study of dystonia in Munich, Germany. Due to favourable referral and treatment patterns in the Munich area, we could provide confident data from dystonia patients seeking botulinum toxin treatment. A total of 230 patients were ascertained, of whom 188 had primary dystonia. Point prevalence ratios were estimated to be 10.1 (95\% confidence interval 8.4-11.9) per 100,000 for focal and 0.3 (0.0-0.6) for generalised primary dystonia. The most common focal primary dystonias were cervical dystonia with 5.4 (4.2-6.7) and essential blepharospasm with 3.1 (2.1-4.1) per 100,000 followed by laryngeal dystonia (spasmodic dysphonia) with $1.0(0.4-1.5)$ per 100,000 .
\end{abstract}

Copyright @2002 S. Karger AG, Basel

\section{Introduction}

Dystonia is defined as a syndrome of sustained involuntary muscle contractions, frequently causing twisting, repetitive movements or abnormal postures [1]. It can affect virtually any part of the body and is classified accordingly. It is also classified by etiology as follows: primary dystonia, where the phenotype is of dystonia alone with the exception that tremor can be present as well; dystonia-plus syndromes, where the clinical phenotypes include other neurologic features in addition to dystonia (at present there are two such dystonia-plus conditions: dopa-responsive dystonia and dystonia-myoclonus); secondary dystonia, resulting from environmental factors (e.g. drugs, stroke, cerebral palsy, tumors), and finally heredodegenerative diseases, which present as a dystoniaplus syndrome with underlying neurodegeneration (e.g. Wilson's disease, Huntington's disease, X-linked dystonia parkinsonism) [1].

Historically, dystonia has been considered to be a rare disorder. The advent of an effective treatment by weakening dystonic muscles with localised injections of botulinum toxin increased clinical interest in the disease and hence recognition. Due to the high costs associated with this therapy, dystonia has become a disease with economic implications in terms of public health [2]. Therefore, epidemiological data can provide important information for cost analysis as well as for the planning of appropriate patient care. However, few epidemiologic studies have been carried out [3-8] (table 1), none so far in Germany. We present service-based data on the point prevalence of dystonia in Munich, Germany, that were collected during a European epidemiological study of dystonia [7, 8].

\begin{tabular}{ll}
\hline KARGER & ( ) 2002 S. Karger AG, Basel \\
0251-5350/02/0214-0202\$18.50/0 \\
$\begin{array}{l}\text { Fax +4161306 1234 } \\
\begin{array}{l}\text { E-Mail karger@karger.ch } \\
\text { www.karger.com }\end{array}\end{array}$ & $\begin{array}{l}\text { Accessible online at: } \\
\text { www.karger.com/journals/ned }\end{array}$
\end{tabular}

PD Dr. Andres O. Ceballos-Baumann

Department of Neurology, Klinikum rechts der Isar

TU München, Möhlstrasse 28

D-81675 Munich (Germany)

Tel. +4989 41404672, Fax +4989 41404867, E-Mail a.ceballos@lrz.tu-muenchen.de 
Table 1. Prevalence ratios per 100,000 from previous studies of dystonia including focal dystonias

\begin{tabular}{|c|c|c|c|c|c|c|}
\hline \multirow[t]{2}{*}{ Location and year of publication } & \multicolumn{6}{|c|}{ Type of dystonia } \\
\hline & generalised & focal & blepharospasm & cervical & writer's cramp & laryngeal \\
\hline Rochester, USA, 1988 [3] & $3.4(0.4-12.4)$ & $29.5(17.2-47.9)$ & $1.7(0.1-9.6)$ & $8.9(2.9-20.7)$ & $6.9(1.9-17.6)$ & $5.2(1.1-15.1)$ \\
\hline Tottori, Japan, 1995 [11] & - & $6.1(3.1-9.2)$ & $1.6^{1}(0.1-3.2)$ & $2.9(0.8-4.9)$ & $1.6(0.1-3.2)$ & - \\
\hline SW Finland, 1996 [6] & - & - & - & $20.9(16.8-25.6)$ & - & - \\
\hline Northern England, 1998 [5] & $1.4(1.0-1.9)$ & $12.9(11.5-14.3)$ & $3.0(2.4-3.7)$ & $6.1(5.2-7.1)$ & $0.7(0.4-1.1)$ & $0.8(0.5-1.3)$ \\
\hline ESDE, $2000[8]$ & - & $11.7(10.8-12.6)$ & $3.6(3.1-4.1)$ & $5.7(5.1-6.4)$ & $1.4(1.1-1.7)$ & $0.7(0.5-0.9)$ \\
\hline
\end{tabular}

Ratio includes both oromandibular and blepharospasm combined.

\section{Methods}

The catchment area for this cross-sectional study was the city limits of Munich. Included were individuals with at least a 3-month history compatible with dystonia-plus signs of dystonia at examination, as defined by Fahn [9], during the period of the study (JanuaryDecember 1996), and residing in the catchment area at the designated prevalence date (June 1, 1996). In Munich, services that provide botulinum toxin treatment are concentrated in the movement disorder clinics of the two universities to which most patients are referred. The two local neurologists and one otolaryngologist-phoniatrician known to carry out treatment with botulinum toxin were asked to participate in this study and provided anonymous information about their patients with dystonia. In Germany, botulinum toxin treatment for dystonia is covered by the health insurances (public and private health insurances) and the social welfare in the rare case of a patient lacking insurance coverage.

Patients who visited the dystonia and botulinum toxin clinics during the period of the study were examined and ascertained, whether this was their first visit or not. In addition, medical records of patients diagnosed with any type of dystonia between 1989 and 1995 were reviewed. Patients identified by these means were contacted and interviewed in order to verify their current clinical and demographic situation.

For each diagnosed case of dystonia, an anonymous data collection sheet was completed. Information was collected on the patients' age and sex, as well as on the type of dystonia. Dystonia was divided into primary dystonia with the following subgroups: dopa-responsive dystonia, paroxysmal kinesigenic dystonia, paroxysmal dystonic choreoathetosis and myoclonic dystonia. For secondary dystonia, the specific cause was recorded using the following categories: tardive dystonia (meaning exposure to neuroleptic or other dopamine receptor-blocking agents for at least 3 months and onset of dystonia within 6 months after discontinuing the drug), cerebrovascular disease, cerebral palsy, cerebral neoplasm, cerebral trauma, Wilson's disease and other causes. The distribution of dystonia was recorded by location. In addition, the year of onset was also recorded to calculate the age at onset.

All data sheets were entered on Microsoft Excel. Means and standard deviations were calculated by standard methods. Comparisons of normally distributed continuous variables were performed using Student's t test. For the calculations of sex ratios, we used the one- sample binomial test. Significance testing for differences between proportions was calculated using the $\chi^{2}$ test. All $\mathrm{p}$ values are twosided and at the $5 \%$ level.

For calculating the point prevalence ratio, the denominator was the entire Munich population. We used the mean of the calculated prevalence for December 1995 and December 1996 (1,322,883). These values were provided by the Department of Statistics of Munich. Confidence intervals were calculated using the Poisson distribution.

\section{Results}

A total of 230 prevalent cases were identified. Two hundred and nine (90.9\%) were seen in one of the university movement disorder clinics, $89 \%$ of them (186 cases) were ascertained during the year 1996 as they visited one of the university clinics and $11 \%$ (23 cases) were acquired by review of medical records. Twenty-one patients $(9.1 \%)$ came from other clinics or practice.

One hundred and eighty-eight cases $(81.7 \%)$ had primary and $42(18.3 \%)$ secondary dystonia. No cases of dopa-responsive, paroxysmal kinesigenic dystonia, paroxysmal dystonic choreoathetosis or myoclonic dystonia were found.

Among the secondary dystonias, 34 cases $(81.7 \%)$ were induced by neuroleptics. Two patients had generalised dystonia due to perinatal damage, 4 developed dystonia after a stroke ( 2 of them had focal and 2 hemidystonia), 1 showed segmental brachial dystonia in a context of cortical-basal ganglionic degeneration and 1 developed focal upper limb dystonia probably due to a local trauma.

The prevalence and sex ratios of primary dystonia subtypes are shown in table 2 . Cervical dystonia was the most common focal primary dystonia, representing more than one half of the cases encountered, followed by blepharo- 
Table 2. The prevalence of primary dystonia in Munich

\begin{tabular}{lclcl}
\hline Type of dystonia & \multirow{2}{*}{$\begin{array}{l}\text { Number } \\
\text { of cases }^{2}\end{array}$} & \multicolumn{2}{l}{ Prevalence $^{3}$} & \multirow{2}{*}{$\begin{array}{l}\text { Male:female } \\
\text { ratio }\end{array}$} \\
\cline { 3 - 4 } & ratio & $95 \% \mathrm{CI}^{3}$ & \\
\hline Blepharospasm & $41(21.8)$ & 3.1 & $2.1-4.1$ & $1: 2.2^{4}$ \\
Cervical & $72(38.3)$ & 5.4 & $4.2-6.7$ & $1: 1.3^{4}$ \\
Laryngeal & $13(6.9)$ & 1.0 & $0.4-1.5$ & $1: 1.3^{4}$ \\
Other focal forms $^{1}$ & $8(4.3)$ & 0.6 & $0.2-1.0$ & $1: 1.0^{4}$ \\
Segmental & $39(20.7)$ & 3.0 & $2.0-3.9$ & $1: 2.3^{4}$ \\
Multifocal & $11(5.9)$ & 0.8 & $0.3-1.3$ & $1: 0.8^{4}$ \\
Generalised & $4(2.1)$ & 0.3 & $0.0-0.6$ & $1: 1.0^{4}$ \\
\hline
\end{tabular}

1 Writer's cramp: 3 males; oromandibular dystonia: 1 female and 1 male; pharyngeal dystonia: 1 female; focal limb dystonia: 2 females.

2 Percentages are shown in parentheses.

3 Crude prevalence ratio per 100,000 .

4 Not statistically significant ( $\chi^{2}$ and binomial test).
Table 3. Age of onset for primary dystonia

\begin{tabular}{lcc}
\hline Type of dystonia & Mean age of onset & SD \\
\hline Blepharospasm & 57.1 & 10.9 \\
Cervical & 41.6 & 14.1 \\
Laryngeal & 48.0 & 11.2 \\
Segmental & 55.0 & 12.8 \\
Multifocal & 39.9 & 23.3 \\
Generalised & 9.8 & 2.9 \\
\hline
\end{tabular}

$\mathrm{SD}=$ Standard deviation

spasm. It is also noted that laryngeal dystonia was about 5 times more prevalent than writer's cramp. The total prevalence of dystonia was 17.4 per 100,000 [95\% confidence interval (CI) 15.1-19.7], for primary dystonia 14.2 (95\% CI 12.2-16.3), for secondary dystonia 3.2 (95\% CI $2.2-$ $4.1)$ and for tardive dystonia 2.6 (95\% CI 1.7-3.4). In the group of primary dystonias, the prevalence of all focal dystonias was 10.1 per 100,000 (95\% CI 8.4-11.9), more than 30 times that of generalised dystonia $(0.3,95 \% \mathrm{CI}$ $0.0-0.6$ ). Table 3 shows the mean age of onset for different types of primary dystonia. As expected, more severe dystonias showed a younger age of onset. We observed a significantly earlier age of onset in males as opposed to females for blepharospasm (51.8 versus 59.6) $(\mathrm{p}=0.04)$. For the other forms of primary dystonia, there was no significant sex difference.

\section{Discussion}

To our knowledge, this study is the first to document the prevalence of dystonia in Germany. The cases have all been diagnosed by experts in the field of movement disorders, using internationally recognised, standardised diagnostic criteria. As part of the Epidemiological Study of Dystonia in Europe (ESDE) Collaborative Group [7, 8], the Munich area has the advantage of active collaboration between the centres providing botulinum toxin treatment. Therefore, due to these favourable referral and treatment patterns, we could provide reliable data from patients treated with botulinum toxin. The results presented here may have implications regarding botulinum toxin clinic service provision [2].

In addition, we evaluate the prevalence of secondary dystonia. Although it is probably underreported here, the figures may provide some idea about this generally unknown prevalence.

Our figures for primary dystonia are similar to those from the ESDE Collaborative Group [8]. This study provides prevalence data for primary dystonia across eight European countries (table 1).

The present study has obviously the methodological limitations of a referral-based survey, as just the diagnosed and referred cases could be ascertained. Patients were mostly referred for botulinum toxin treatment. This may have led to an increased proportion of focal and segmental dystonias and at the same time to the underreporting of generalised and multifocal dystonias, since these patients benefit less from a botulinum toxin therapy than 
those with focal dystonia. Likewise, most patients with generalised dystonia have childhood onset. Pediatric patients were only rarely referred to adult neurological practices for botulinum toxin which at the time of the study was not used by neuropediatricians. These problems may also explain why we did not ascertain any case of doparesponsive dystonia. However, the clinical presentation of dopa-responsive dystonia is very variable and often misdiagnosed [10].

The prevalence rates of primary dystonia are lower than those reported by Nutt et al. [3], which were based on the records of the Mayo Clinic, Rochester, Minn., USA, but similar to those by Duffey et al. [5], except for generalised dystonia (table 1). Duffey et al. [5] performed a community-based study in the northeast of England that included a number of complementary methods, such as an awareness-raising campaign, recruitment of patients from dystonia self-help groups, as well as a postal survey in a restricted area.

Compared with our data, Nakashima et al. [11] reported a lower prevalence of focal dystonias in the western part of Tottori Prefecture in Japan. These authors suggested that genetic factors may account for the low prevalence compared with the study of Nutt et al. [3]. In contrast, the prevalence ratio for cervical dystonia in southwestern Finland reported by Erjanti et al. [6] was more than twice as high as the ratio observed in our study and in Rochester.

The true prevalence of dystonia is difficult to estimate. There are several reasons for this: as family studies have shown [12, 13], dystonia is underreported, since many patients with mild symptoms are not aware of their condition. Dystonia is also frequently undiagnosed. In their series, Butler et al. [14] reported that a total of $66.7 \%$ of patients needed at least five consultations before diagnosis was achieved and $65.7 \%$ were misdiagnosed at some stage. Finally, as all studies looking at the prevalence of focal dystonias are service-based rather than communitybased, except the study by Duffey et al. [5], the comparability of the centre estimates is problematic. The differences in ratios could be explained by ascertainment and selection bias, but may also reflect an underlying environmental or genetic factor. We identified 42 patients with secondary dystonia. We have probably missed the cases due to metabolic or structural disorders present in childhood. Nevertheless, our proportions are strikingly similar to those reported by Fahn et al. [15]. In their series, secondary dystonia represented $23 \%$ of all the cases: $12 \%$ of focal dystonia, $14 \%$ of segmental, $46 \%$ of multifocal and $42 \%$ of generalised dystonia. In our series, $18.3 \%$ of the cases are secondary dystonia, accounting for $7 \%$ of focal dystonia, $35 \%$ of segmental, $38 \%$ of multifocal and $33 \%$ of generalised dystonia.

The most common cause of secondary dystonia in the present study was exposure to neuroleptics in 34 cases, yielding a prevalence ratio of 2.6 per 100,000 . The given prevalence of tardive dystonia in the neuroleptic-treated population ranges from 0.4 to $21 \%$ [16]. However, it is likely that many of the tardive dystonia patients are not referred to neurology clinics, as the prevalence is so high in long-term psychiatric institutions. In agreement with other reports $[3,5,8,17]$, the most common type of focal dystonia was cervical dystonia, followed by blepharospasm with prevalence ratios of 5.4 and 3.1 per 100,000 , respectively. However, our estimate for writer's cramp differs from the series of Nutt et al. [3], Soland et al. [17], Duffey et al. [5] and the ESDE Collaborative Group [8] where writer's cramp accounted for $21,19,6$ and $12 \%$ of focal dystonias, respectively. We found that writer's cramp just accounted for $2.2 \%$ of our focal dystonia patients, yielding a prevalence of 0.2 per 100,000 (95\% CI 0.0-0.5). Nevertheless, taking the CIs into account, our figures are again comparable with those from Duffey et al. [5] (0.7 per 100,000, 95\% CI 0.4-1.1).

Comparing the mean age of onset in men and women, we found a significantly earlier age in males for blepharospasm (51.8 versus 59.6). Sex differences depending on the age of onset have also been recently reported in the ESDE study [7] for primary focal and segmental dystonia. This study observed a significantly earlier age of onset for cervical dystonia, blepharospasm, laryngeal dystonia and segmental dystonia in males, while for writer's cramp and focal limb dystonia, this trend was reversed.

In summary, as service-based study, our figures may underestimate the true prevalence of dystonia, but due to the characteristics of patient ascertainment, it reflects the point prevalence of patients with dystonia seeking botulinum toxin treatment.

\section{Acknowledgments}

The authors gratefully acknowledge the statistical advice of $\mathrm{R}$. Busch. Specific acknowledgment should go to Dr. P. Franz, Dr. K.O. Sigel and Dr. K. Joussen for providing anonymous information about their dystonia patients. 


\section{References}

1 Fahn S, Bressman SB, Marsden CD: Classification of dystonia; in Fahn S, Marsden CD, DeLong MR (eds): Dystonia 3: Advances in Neurology. Philadelphia, Lippincott, 1998, vol 78, pp $1-11$.

2 Dodel RC, Kirchner A, Koehne-Volland R, Kunig G, Ceballos-Baumann A, Naumann M, Brashear A, Richter HP, Szucs TD, Oertel WH: Costs of treating dystonias and hemifacial spasms with botulinum toxin A. Pharmacoeconomics 1997;12:695-706.

3 Nutt JG, Muenter MD, Aronson A, Kurland LT, Melton LJ: Epidemiology of focal and generalized dystonia in Rochester, Minnesota. Mov Disord 1988;3:188-194.

4 Claypool DW, Duane DD, Ilstrup DM, Melton LJ 3rd: Epidemiology and outcome of cervical dystonia (spasmodic torticollis) in Rochester, Minnesota. Mov Disord 1995;10:608-614.

5 Duffey POF, Butler AG, Hawthorne MR Barnes MP: The epidemiology of the primary dystonias in the North of England; in Fahn S, Marsden CD, De Long MR (eds): Dystonia 3: Advances in Neurology. Philadelphia, Lippincott, 1998, vol 78, pp 121-125.
6 Erjanti HM, Martilla RJ, Rinne UK: The prevalence and incidence of cervical dystonia in south-western Finland. Mov Disord 1996; 11(suppl 1):215.

7 The Epidemiological Study of Dystonia in Europe (ESDE) Collaborative Group: Sex-related influences on the frequency and age of onset of primary dystonia. Neurology 1999;10:18711873.

8 The Epidemiological Study of Dystonia in Europe (ESDE) Collaborative Group: A prevalence study of primary dystonia in eight European countries. J Neurol 2000;247:787-792.

9 Fahn S: Concept and classification of dystonia; in Fahn S, Marsden CD, Calne DB (eds): Dystonia 2: Advances in Neurology. Philadelphia, Lippincott, 1988, vol 50, pp 1-8.

10 Bandmann O, Marsden CD, Wood NW: Atypical presentations of dopa-responsive dystonia; in Fahn S, Marsden CD, De Long MR (eds): Dystonia 3: Advances in Neurology. Philadelphia, Lippincott-Raven, 1998, vol 78, pp 283290.

11 Nakashima K, Kusumi M, Inoue Y, Takahashi $\mathrm{K}$ : Prevalence of focal dystonias in the western area of Tottori Prefecture in Japan. Mov Disord 1995;10:440-443.
12 Waddy HM, Fletcher NA, Harding AE, Marsden CD: A genetic study of idiopathic focal dystonias. Ann Neurol 1991;29:320-324.

13 Fletcher NA, Harding AE, Marsden CD: A genetic study of idiopathic torsion dystonia in the United Kingdom. Brain 1990;113:379395.

14 Butler AG, Duffey POF, Hawthorne MR Barnes MP: The socioeconomic implications of dystonia; in Fahn S, Marsden CD, De Long MR (eds): Dystonia 3: Advances in Neurology. Philadelphia, Lippincott-Raven, 1998, vol 78, pp 349-358.

15 Fahn S, Marsden CD, Calne DB: Classification and investigation of dystonia; in Marsden CD, Fahn S (eds): Movement Disorders 2. London, Butterworth, 1987, pp 332-353.

16 Adityanjee MD, Aderibigbe YA, Jampala VC, Mathews T: The current status of tardive dystonia. Biol Psychiatry 1999;45:715-730.

17 Soland VL, Bathia KP, Marsden CD: Sex prevalence of focal dystonias. J Neurol Neurosurg Psychiatry 1996;60:204-205. 